\title{
PREFACE
}

\section{Environmental Antibiotics and Antibiotic Resistance: From Problems to Solutions}

\author{
Xin Yu $(\bowtie)^{1}$, Virender K. Sharma $(\bowtie)^{2}$, Hui Li $(\bowtie)^{3}$ \\ 1 Institute of Urban Environment, Chinese Academy of Sciences, Xiamen 361021, China \\ 2 Department of Environmental Health, School of Public Health, Texas A\&M University, College Station, TX 77843, USA \\ 3 Department of Plant, Soil and Microbial Sciences, Michigan State University, East Lansing, MI 48824, USA
}

(C) Higher Education Press and Springer-Verlag GmbH Germany, part of Springer Nature 2019

Nowadays, the international academic community and the public regard both antibiotics and antibiotic resistance bacteria/ genes as emerging contaminants. These contaminants are increasing to pose severe threats to human health and to the sustainable development of livestock production. In 2014, World Health Organization (WHO) issued "antimicrobial resistance global report on surveillance". The report seriously pointed it out that "in a post-antibiotic era - in which common infections and minor injuries can kill - far from being an apocalyptic fantasy, is instead a very real possibility for the 21 st century".

Different from their clinical counterparts, the environmental antibiotics and antibiotic resistance occur in matrices that are more complicated and are also impacted by much more physical, chemical, biological and combined factors. This complexity makes it difficult to elucidate their occurrence, transportation, transformation, and fate in the environmental compartments.

Although this theme has become one of the research hotspots in environmental science and engineering, many scientific and technical questions remain unanswered. For example, it is in pivotal to develop and apply novel analysis tools for measuring both antibiotics and their resistance due to their trace levels and/or instability in the complicated environmental media. Their acute and chronic effects on individual organism, population, and ecosystem levels should be deeply investigated. The gap between environmental and clinical studies should be reduced to assess their risks appropriately. In addition, novel and effective treatment technologies are of great practical significance for building up the sustainable antibiotic and antibiotic resistance management system.

In this special issue, we attempted to address the challenges by clarifying the problems and find out the preliminary solutions of antibiotics and antibiotic resistance. The special issue presents methodological aspects and the molecular approaches like conventional qPCR and high-throughput qPCR commonly used in the investigations. The X-omics are discussed and summarized on characterization of antibiotic resistome. On the environmental behaviors of antibiotics and antibiotic resistance bacteria/genes, the interaction between adverse factors such as heavy metals and resistant bacteria, investigations on ARGs diversity in specific inhabits and subsequently treatment processes are given. For example, the ARGs in a dialysis water treatment system, an extremely oligotrophic environment, was detected and quantified, which revealed the health risks that were neglected previously. On effective removal methods, a variety of conventional and novel technologies have been reported including composting, anaerobic digestion, chlorination, and dielectric barrier discharge, and UV based treatment. However, much more research is critically needed to establish the relationship between the environmental and clinical resistant bacteria. Hopefully, this special issue will stimulate advances in this direction in the near future.

We thank all authors, reviewers, and editorial staff of FESE for their efforts and contributions to this special issue.

Received June 5, 2019

$\square$ Corresponding authors

E-mail: xyu@iue.ac.cn (X. Yu); vsharma@sph.tamhsc.edu (V. Sharma); lihui@msu.edu (H. Li)

Special Issue - Environmental Antibiotics and Antibiotic Resistance (Responsible Editors: Xin Yu, Hui Li \& Virender K. Sharma) 